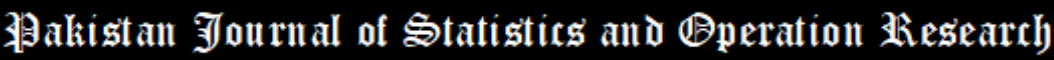

\section{The McDonald Lindley-Poisson Distribution}

\author{
Ana Percontini ${ }^{1}$, Frank Gomes-Silva ${ }^{2}$, Ronaldo V. da Silva ${ }^{3}$, \\ Laba Handique ${ }^{4,}$, Pedro Rafael D. Marinho ${ }^{5 *}$ \\ ${ }^{*}$ Corresponding author
}

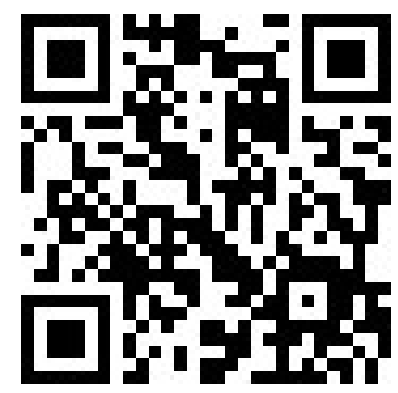

1. Department of Exact Sciences, State University of Feira de Santana, Feira de Santana, BA, Brazil, anappaixao@gmail.com

2. Department of Statistics and Informatics, Federal Rural University of Pernambuco, Recife, PE, Brazil, franksinatrags@gmail.com

3. Recife Military School, Recife, PE, Brazil, ronaldovenanciorvs@gmail.com

4. Departament of Statistics, Dibrugarh University, Dibrugarh, India, handiquelaba@gmail.com

5. Department of Statistics, Federal University of Paraíba, João Pessoa, PB, Brazil, pedro.rafael.marinho@gmail.com

\begin{abstract}
We propose the McDonald Lindley-Poisson distribution and derive some of its mathematical properties including explicit expressions for moments, generating and quantile functions, mean deviations, order statistics and their moments. Its model parameters are estimated by maximum likelihood. A simulation study investigates the performance of the estimates. The new distribution represents a more flexible model for lifetime data analysis than other existing models as proved empirically by means of two real data sets.
\end{abstract}

Key Words: Lindley-Poisson distribution; Maximum likelihood estimation; McDonald distribution; Moment; Monte Carlo simulation.

Mathematical Subject Classification: 62E10, 62E15.

\section{Introduction}

The Poisson distribution has been used to generate several flexible continuous distributions by compounding methods for modeling survival data. Many generalizations based on the Poisson distribution are investigated in recent years. For example, the Conway-Maxwell-Poisson discussed in Minka et al. (1) and Shmueli et al. (2), exponential Poisson proposed by Kus (3), Weibull Poisson studied by Hemmati et al. (4) and exponentiated Burr XII Poisson proposed and studied by Silva et al. (5).

Further, the Lindley distribution was introduced by Lindley (6) to illustrate the difference between fiducial and posterior distributions. Ghitany et al. (7) investigated the properties of the latter distribution and showed that it is a better model than the exponential distribution. The Lindley has also some advantages since to its hazard rate function (hrf) can exhibit bathtub shapes, and then it becomes more versatile and flexible for compounding with other distributions.

Sankaran (8) introduced the Poisson-Lindley distribution by assuming that the Poisson parameter follows a Lindley distribution. Mahmoudi and Zakerzadeh (9) generalized the Poisson-Lindley distribution and showed that their generalization has more flexibility in analyzing count data. Bhati et al. (10) proposed a 
generalized Poisson-Lindley distribution which can be unimodal and over-dispersed. Further, the LindleyPoisson (LP), a sub-model of the exponentiated power Lindley Poisson, was investigated by Pararai et al. (11).

Modeling real life data is very useful in many applied areas and considerable efforts have been done to construct new distributions for survival data. However, there still remain many problems involving real data, which are not contemplated by existing distributions. Researchers in recent years proposed new distributions by extending the exponential, gamma and Weibull, among others. Some of these generalizations have been used extensively for modeling data in several areas such as the actuarial, engineering, biological studies, economics, finance and medical sciences. However, extra works are needed for extended forms of these distributions in many applied areas of lifetime analysis.

For an arbitrary parent cumulative distribution function (cdf) $G(x ; \boldsymbol{\tau})$ with parameter vector $\boldsymbol{\tau}$ and $g(x ; \boldsymbol{\tau})=$ $\frac{d G(x ; \boldsymbol{\tau})}{d x}$, the probability density function (pdf) of the McDonald class ("Mc-G" for short) proposed by Alexander et al. (12) is

$$
f(x ; a, b, c, \boldsymbol{\tau})=\frac{c}{B(a, b)} g(x ; \boldsymbol{\tau}) G^{a c-1}(x ; \boldsymbol{\tau})\left[1-G^{c}(x ; \boldsymbol{\tau})\right]^{b-1}, \quad x \in \mathbb{R},
$$

where $a>0, b>0$ and $c>0$ are additional shape parameters which introduce skewness and provide greater flexibility of the tails of $f(x)$.

The class (1) includes two important family models: the beta generalized family (Eugene et al. (13)) when $c=1$ and Kumaraswamy generalized family (Cordeiro and Castro, (14)) when $a=1$. The Mc-G distribution with baseline $G(x ; \boldsymbol{\tau})$ is simply the beta generalized distribution with baseline $G(x ; \boldsymbol{\tau})^{c}$. This simple transformation may facilitate the computation of several of its properties. For $G(x ; \boldsymbol{\tau})=x$, we obtain the McDonald distribution and the beta and Kumaraswamy distributions follow as special cases when $c=1$ and $a=1$, respectively. The cdf corresponding to (1) is

$$
F(x)=I_{G^{c}(x ; \boldsymbol{\tau})}(a, b)=\frac{1}{B(a, b)} \int_{0}^{G^{c}(x ; \boldsymbol{\tau})} \omega^{a-1}(1-\omega)^{b-1} \mathrm{~d} \omega,
$$

where $I_{x}(a, b)=B(a, b)^{-1} \int_{0}^{x} \omega^{a-1}(1-\omega)^{b-1} \mathrm{~d} \omega$ denotes the incomplete beta function ratio.

The random variable $X$ with pdf $(1)$ is $X=Q_{G}\left(V^{1 / c} ; \boldsymbol{\tau}\right)$, where $x=Q_{G}(u ; \boldsymbol{\tau})=G^{-1}(u ; \boldsymbol{\tau})$ is the quantile function (qf) of $\mathrm{G}$ and $V$ has a beta distribution with parameters $a$ and $b$. One important characteristic of the Mc-G class is its ability to fit skewed data that cannot be properly fitted by existing distributions.

In this paper, we study the McDonald Lindley-Poisson (McLP) distribution, which extends the Kumaraswamy Lindley-Poisson explored and studied by Pararai et al. (11). The motivation to study the McLP distribution is because of the extensive use of Lindley distribution in survival analysis and the fact that existing generalizations can be improved. Some important contributions of this paper are:

- The proposed distribution overcomes a limitation of its baseline, whose hazard function presents only monotonic increasing and upside-down bathtub shapes. The McLP hrf admits the five main characteristics: monotonically increasing, monotonically decreasing, bathtub, upside-down bathtub and sigmoid shapes.

- An advantage of fitting a wider model to real data is that we can verify, using the likelihood ratio (LR) statistics, whether its sub-models with fewer parameters, can be more proper to the data. The McLP distribution extends eight lifetime distributions, including the beta LP (BLP) and exponentiated LP (ELP) models. The BLP distribution becomes a very competitive model to some well-known fourparameter lifetime distributions such as the Burr XII Poisson, beta inverse Weibull, beta exponentiated Lindley and ELP.

- Although the proposed model has five parameters, it can provide better fits, based on Anderson-Darling and Cramér-von Mises statistics, than ones with less number of parameters.

The paper is organized as follows. In Section 2, we define the McLP distribution and highlight some special cases. In Section 3, we demonstrate that the McLP density function is a linear combination of LP densities. Some mathematical properties of the McLP distribution are also obtained in this section including ordinary 
and incomplete moments, mean deviations and generating and quantile functions, order statistics and their moments. The estimation of the model parameters by maximum likelihood is addressed in Section 4. In Section 5, we perform a simulation study to verify the adequacy of the estimates. Two applications to real data illustrate the flexibility of the new distribution in Section 6. Section 7 offers some concluding remarks.

\section{The McLP distribution}

Suppose that $Z$ has a truncated Poisson distribution with parameter $\theta>0$ and probability mass function

$$
p(z ; \theta)=\frac{\theta^{z} \mathrm{e}^{-\theta}}{\left(1-\mathrm{e}^{-\theta}\right) z !}, z=1,2, \ldots
$$

We consider independent and identically distributed (iid) random variables $\left\{W_{i}\right\}_{i=1}^{Z}$ having Lindley density function

$$
\pi(w)=\frac{\beta^{2}}{\beta+1}(1+w) \mathrm{e}^{-\beta w}, w>0
$$

where $\beta>0$ is the scale parameter.

Assuming that $W$ 's and $Z$ are independent random variables, $X=\min \left\{W_{1}, \ldots, W_{Z}\right\}$ defines the LP distribution. The pdf of $X$ takes the form (for $x>0$ )

$$
g(x ; \beta, \theta)=\frac{\theta \beta^{2}(1+x) \mathrm{e}^{-\beta x} \exp \left\{\theta\left[1-\left(1+\frac{\beta x}{\beta+1}\right)\right]\right\}}{(\beta+1)\left(\mathrm{e}^{\theta}-1\right)} .
$$

The cdf corresponding to $(3)$ is

$$
G(x ; \beta, \theta)=\frac{1-\exp \left\{\theta\left[1-\left(1+\frac{\beta x}{\beta+1}\right) \mathrm{e}^{-\beta x}\right]\right\}}{1-\mathrm{e}^{\theta}} .
$$

The LP model is well-motivated for biological studies. For example, consider the time to relapse of cancer under the first-activation scheme. Suppose that the number of carcinogenic cells for an individual left active after the initial treatment follows a truncated Poisson distribution and let $W_{i}$ be the time spent for the $i$ th carcinogenic cell to produce a detectable cancer mass (for $i \geq 1$ ). If $\left\{W_{i}\right\}_{i \geq 1}$ is a sequence of iid Lindley random variables independent of $Z$, then the time to relapse of cancer of a susceptible individual can be modeled by the LP distribution.

The five-parameter McLP distribution is defined from (1) by taking $G(\cdot)$ and $g(\cdot)$ to be the cdf and pdf of the LP distribution. Its density function is (for $x>0$ )

$$
f(x ; a, b, c, \beta, \theta)=\frac{c \theta \beta^{2}(1+x) \mathrm{e}^{-\beta x} \mathrm{e}^{\varphi(x)}}{B(a, b)(\beta+1)\left(\mathrm{e}^{\theta}-1\right)}\left[\frac{1-\mathrm{e}^{\varphi(x)}}{1-\mathrm{e}^{\theta}}\right]^{a c-1}\left\{1-\left[\frac{1-\mathrm{e}^{\varphi(x)}}{1-\mathrm{e}^{\theta}}\right]^{c}\right\}^{b-1}
$$

where $\varphi(x)=\theta\left[1-\left(1+\frac{\beta x}{\beta+1}\right) \mathrm{e}^{-\beta x}\right], \theta>0, \beta>0$ is a scale parameter, $a>0, b>0$ and $c>0$ are shape parameters. Henceforth, the McLP distribution is denoted by the random variable $X \sim \operatorname{McLP}(a, b, c, \beta, \theta)$. According to Gui et al. (15), the LP distribution has only monotonically increasing or upside-down bathtub hazard rates. On the other hand, as we shall see later, the hrf of the McLP distribution allows for monotonically increasing, monotonically decreasing, bathtub, upside-down bathtub and sigmoid shapes.

The density function (4) includes as special models some distributions. In fact, the LP distribution is a basic exemplar when $a=b=c=1$. The BLP distribution is obtained when $c=1$. For $a=1$, we have the Kumaraswamy LP (KwLP) distribution. The McLP distribution becomes the ELP when $a=b=1$. In addition, we obtain the exponentiated Lindley distribution when $\theta \rightarrow 0^{+}$.

The cdf and hrf of $X$ are, respectively,

$$
F(x ; a, b, c, \beta, \theta)=I_{G^{c}(x ; \beta, \theta)}(a, b)=\frac{1}{B(a, b)} \int_{0}^{G^{c}(x ; \beta, \theta)} \omega^{a-1}(1-\omega)^{b-1} \mathrm{~d} \omega
$$


and

$$
\begin{aligned}
h(x ; a, b, c, \beta, \theta) & =\frac{c \theta \beta^{2}(1+x) \mathrm{e}^{\varphi(x)-\beta x}}{(\beta+1)\left(\mathrm{e}^{\theta}-1\right) B(a, b)\left\{1-I_{G^{c}(x)}(a, b)\right\}}\left[\frac{1-\mathrm{e}^{\varphi(x)}}{1-\mathrm{e}^{\theta}}\right]^{a c-1} \\
& \times\left\{1-\left[\frac{1-\mathrm{e}^{\varphi(x)}}{1-\mathrm{e}^{\theta}}\right]^{c}\right\}^{b-1} .
\end{aligned}
$$

Some possible shapes of the density function (4) and hrf (6) for selected parameter values are displayed in Figures 1 and 2, respectively.

The random variable $X$ with cdf (5) has a simple stochastic representation $X=Q_{G}\left(V^{1 / c}\right)$, where $x=$ $Q_{G}(u)=G^{-1}(u)$ denotes the qf corresponding to $G$ and $V$ has a beta distribution with parameters $a$ and $b$. The McLP distribution can be simulated by inverting Equation (5) as follows:

If $V$ is a beta random variable with parameter $a$ and $b$, then

$$
X=\frac{\beta\left\{1-(1-V)^{1 / \theta}\right\}}{(1-V)^{1 / \theta}}
$$

follows the $\operatorname{McLP}(a, b, c, \beta, \theta)$ distribution. This method is useful because of the existence of strong generators for beta random variables. 

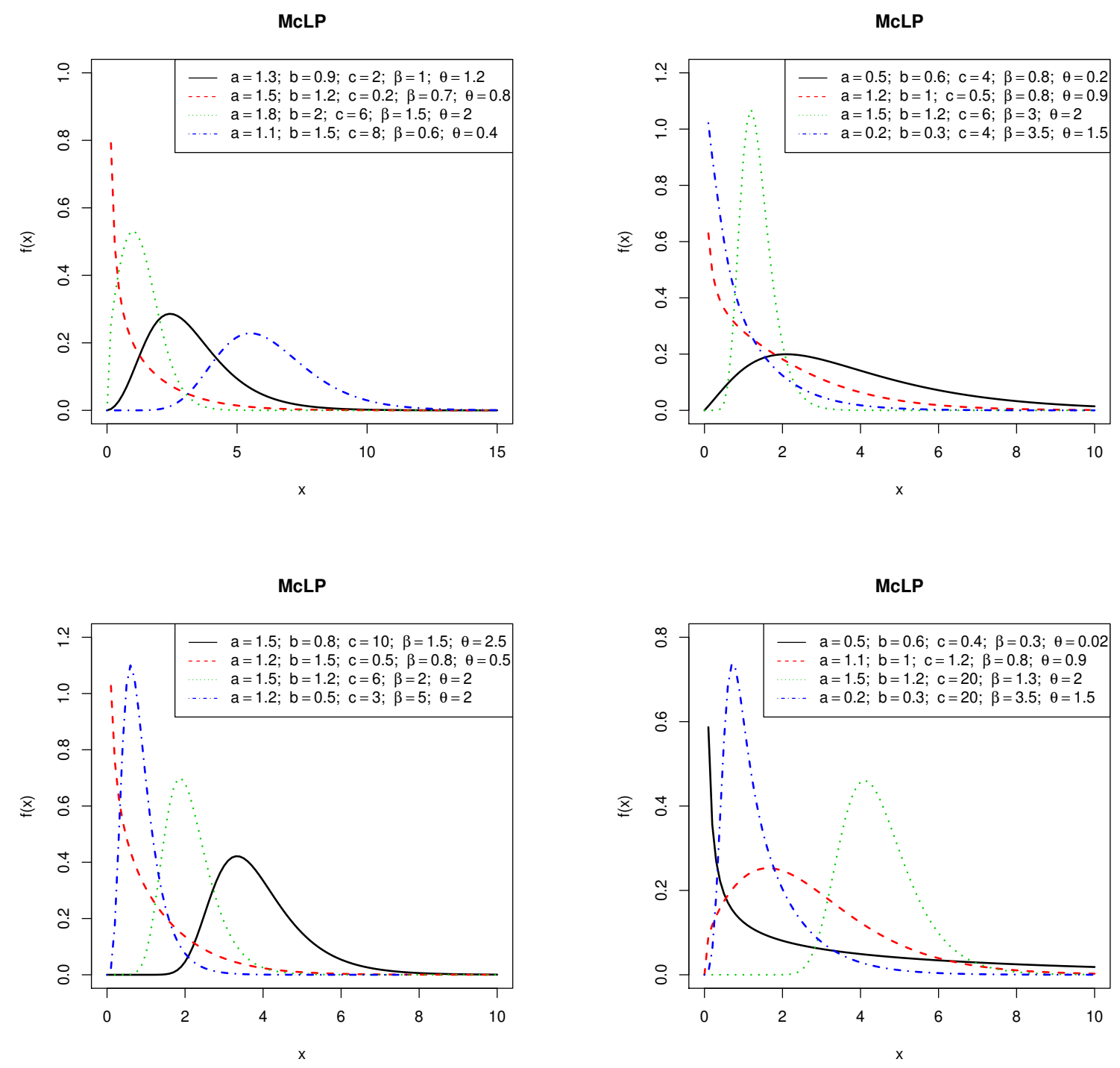

Figure 1: Plots of the McLP density function for some parameter values. 

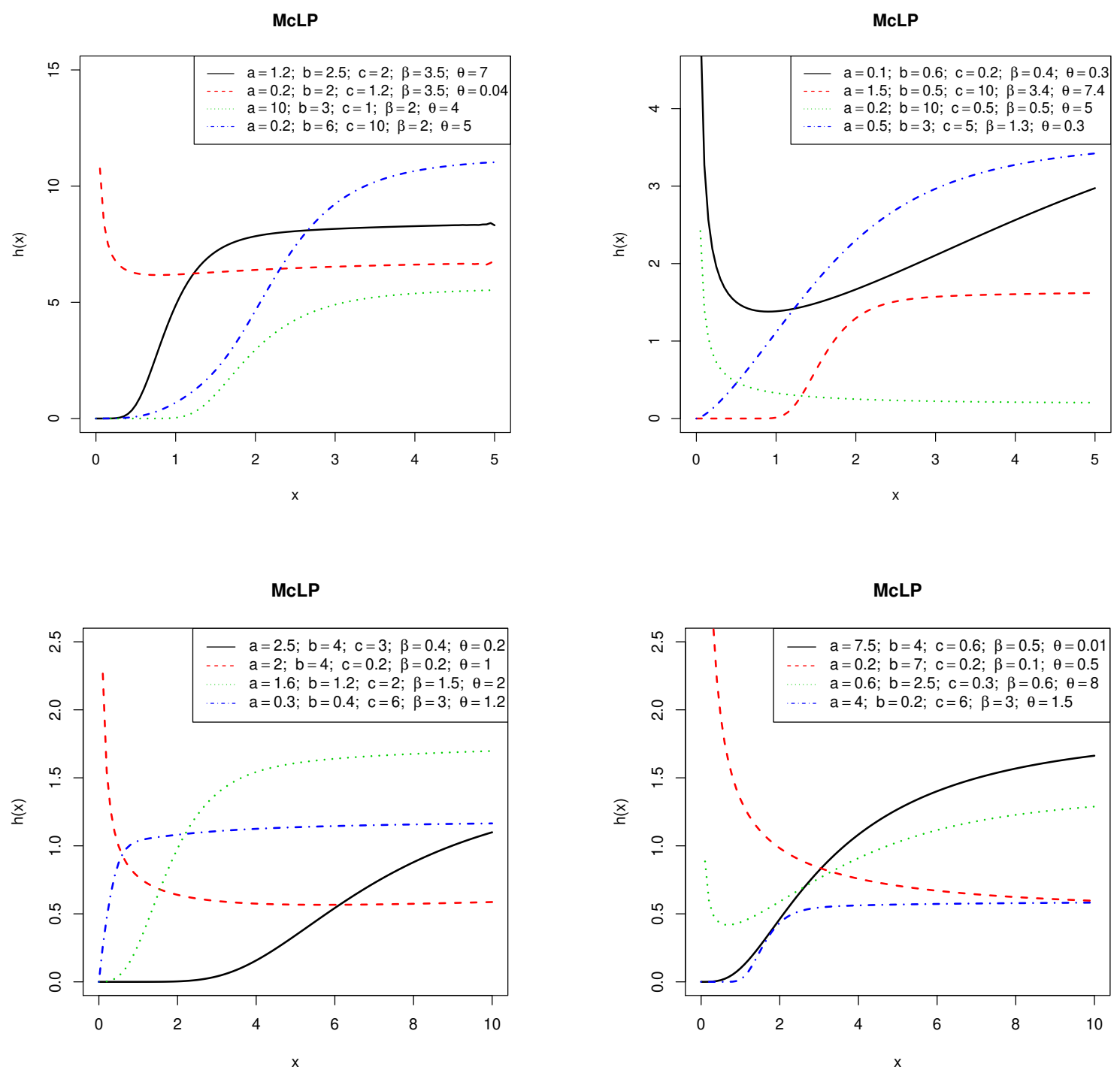

Figure 2: Plots of the McLP hrf for some parameter values.

\section{Properties of the new distribution}

\subsection{Quantile function}

The qf of the McLP distribution, say $Q(u)=F^{-1}(u)$, can be expressed in terms of the beta qf. By inverting (5), we can write

$$
X=Q(u)=-1-\frac{1}{\beta}-\frac{1}{\beta} W_{-1}\left((\beta+1) \mathrm{e}^{-(\beta+1)}\left\{\log \left[1-z^{1 / c}\left(1-\mathrm{e}^{\theta}\right)\right]^{1 / \theta}-1\right\}\right)
$$

where $W_{-1}(\cdot)$ is the negative branch of the Lambert function (see Corless et al. $(16)$ ), and $z=Q_{a, b}(u)=$ $I_{u}^{-1}(a, b)$ denotes the beta qf with parameters $a$ and $b$. In the Wolfram website (http://functions.wolfram.com/06.23.06.0004.01 
can be found the following expansion for the beta qf

$$
Q_{a, b}(u)=\sum_{i=1}^{4} A_{i}[B(a, b) a u]^{i / a}+O\left(u^{5 / a}\right),
$$

where the quantities $A_{i}(i=1,2,3,4)$ are

$$
A_{1}=1, \quad A_{2}=\frac{b-1}{a+1}, \quad A_{3}=\frac{(b-1)\left[a^{2}+a(3 b-1)+5 b-4\right]}{2(a+1)^{2}(a+2)}
$$

and

$$
A_{4}=\frac{(b-1)\left[a^{4}+(6 b-1) a^{3}+(b+2)(8 b-5) a^{2}\right]}{(3 a+1)^{3}(a+2)(a+3)}
$$

\subsection{Linear representation}

In this section, we derive a useful linear representation for the McLP density function. If $|z|<1$ and $d>0$ is a real non-integer, the power series holds

$$
(1-z)^{d-1}=\sum_{k=0}^{\infty}(-1)^{k}\left(\begin{array}{c}
d-1 \\
k
\end{array}\right) z^{k}
$$

where the binomial coefficient is defined for any real. Based on Equation (8) and by expanding the binomial in (1), we have

$$
f(x)=\frac{c}{B(a, b)} g(x) \sum_{k=0}^{\infty}(-1)^{k}\left(\begin{array}{c}
b-1 \\
k
\end{array}\right) G^{(k+a) c-1}(x)
$$

and

$$
f(x)=\sum_{k=0}^{\infty} \omega_{k} h_{(k+a) c}(x)
$$

where the weights are $\omega_{k}=\frac{(-1)^{k}\left(\begin{array}{c}b-1 \\ k\end{array}\right)}{(k+a) B(a, b)}, k \geq 0$, and $h_{(k+a) c}(x)$ has the exponentiated- $G$ (Exp-G) density with power parameter $(k+a) c$, say Exp- $G[(k+a) c]$. If $Y_{(k+a) c} \sim \operatorname{Exp}-G[(k+a) c]$, its pdf and cdf are $h_{(k+a) c}(y)=(k+a) c g(y) G^{(k+a) c-1}(y)$ and $H_{(k+a) c}(y)=G^{(k+a) c}(y)$, respectively.

Based on Equation (9) and using the pdf and cdf of the Exp-G distribution, the McLP density can be expressed as

$$
f(x)=\sum_{k=0}^{\infty} \frac{c \omega_{k}(k+a) \theta \beta^{2}(1+x) \mathrm{e}^{-\beta x} \mathrm{e}^{\varphi(x)}\left[1-\mathrm{e}^{\varphi(x)}\right]^{(k+a) c-1}}{(\beta+1)\left(1-\mathrm{e}^{\theta}\right)^{(k+a) c-1}\left(\mathrm{e}^{\theta}-1\right)},
$$

which leads to a linear representation

$$
f(x)=\sum_{j=0}^{\infty} q_{j} g\left(x ; \beta, \theta_{j}\right), \quad x>0
$$

where $\theta_{j}=(j+1) \theta>0, g\left(x ; \beta, \theta_{j}\right)$ denotes the LP density and the weights are

$$
q_{j}=\frac{c \mathrm{e}^{\theta j}\left(1-\mathrm{e}^{\theta j}\right)^{-1}}{(\beta+1)(j+1) ! B(a, b)} \sum_{k=0}^{\infty} \frac{(-1)^{k+j}\left(\begin{array}{c}
b-1 \\
k
\end{array}\right) \Gamma[(k+a) c]}{\left(1-\mathrm{e}^{\theta}\right)^{(k+a) c-1} \Gamma[(k+a) c-j]} .
$$

By integrating (10), we have

$$
F(x)=\sum_{j=0}^{\infty} q_{j} G\left(x ; \beta, \theta_{j}\right), \quad x>0,
$$

where $G\left(x ; \beta, \theta_{j}\right)$ denotes the cdf of the LP distribution. 
Some mathematical properties of the McLP distribution can be determined directly from (10) using the properties of the LP distribution. These properties can be found analytically in softwares such as MATHEMATICA, MATLAB and MAPLE since these computing softwares have the ability to deal with most complex analytical expressions.

\subsection{Moments}

In this section, we obtain the moments of the McLP distribution. Some of the most important features and characteristics of a distribution can be studied through moments (e.g., tendency, dispersion, skewness and kurtosis). The $n$th ordinary moment of $X$ comes from Equation (10) as

$$
\begin{aligned}
\mu_{n}^{\prime}=E\left(X^{n}\right) & =\sum_{j=0}^{\infty} q_{j} \int_{0}^{\infty} x^{n} g\left(x ; \beta, \theta_{j}\right) \mathrm{d} x \\
& =\sum_{j=0}^{\infty} \frac{q_{j} \theta \beta^{2}}{\left(\mathrm{e}^{\theta_{j}}-1\right)(\beta+1)} \int_{0}^{\infty} x^{n}(1+x) \mathrm{e}^{-\beta x} \\
& \times \exp \left\{\theta_{j}\left[1-\left(1+\frac{\beta x}{\beta+1}\right) \mathrm{e}^{-\beta x}\right]\right\} \mathrm{d} x
\end{aligned}
$$

By using the power series for the exponential function, we obtain

$$
\begin{aligned}
\mu_{n}^{\prime} & =\sum_{j, i=0}^{\infty} \sum_{m=0}^{i} \frac{(-1)^{m} q_{j} \theta_{j}^{i+1} \beta^{2}\left(\begin{array}{c}
i \\
m
\end{array}\right)}{i !(\beta+1)\left(\mathrm{e}^{\theta_{j}}-1\right)} \\
& \times \int_{0}^{\infty} x^{n}(1+x) \mathrm{e}^{-\beta x(1+m)}\left(1+\frac{\beta x}{\beta+1}\right)^{m} \mathrm{~d} x
\end{aligned}
$$

By using the binomial expansion, (12) can be rewritten as

$$
\begin{aligned}
\mu_{n}^{\prime} & =\sum_{j, i=0}^{\infty} \sum_{m=0}^{i} \sum_{s=0}^{m} \frac{(-1)^{m} q_{j} \theta_{j}^{i+1} \beta^{2+s}\left(\begin{array}{c}
i \\
m
\end{array}\right)\left(\begin{array}{c}
m \\
s
\end{array}\right)}{i !(\beta+1)^{1+s}\left(\mathrm{e}^{\theta_{j}}-1\right)} \\
& \times \int_{0}^{\infty} x^{n+s}(1+x) \mathrm{e}^{-\beta x(1+m)} \mathrm{d} x .
\end{aligned}
$$

The last integral can be computed using the MATHEMATICA software. Then,

$$
\mu_{n}^{\prime}=\sum_{i=0}^{\infty} \sum_{m=0}^{i} \sum_{s=0}^{m} \gamma_{i, m, s}[1+\beta(1+m)+n+s] \Gamma(1+n+s),
$$

where

$$
\gamma_{i, m, s}=\sum_{j=0}^{\infty} \frac{(-1)^{m} q_{j} \beta^{-n} \theta_{j}^{i+1}(1+m)^{-2-n-s}\left(\begin{array}{c}
i \\
m
\end{array}\right)\left(\begin{array}{c}
m \\
s
\end{array}\right)}{i !(\beta+1)^{1+s}\left(\mathrm{e}^{\theta_{j}}-1\right)},
$$

$\Gamma(z)$ is the gamma function and $\Gamma(a, z)=\int_{z}^{\infty} x^{a-1} \mathrm{e}^{-x} \mathrm{~d} x(z>0)$ denotes the incomplete gamma function.

The incomplete moments of $X$ can be expressed in terms of the incomplete moments of the LP distribution 
from Equation (10). We obtain

$$
\begin{aligned}
m_{n}(y) & =E\left(X^{n} \mid X<y\right)=\sum_{j=0}^{\infty} q_{j} \int_{0}^{y} x^{n} g\left(x ; \beta, \theta_{j}\right) \mathrm{d} x \\
& =\sum_{j, s, i=0}^{\infty} \sum_{m=0}^{i} \frac{(-1)^{m+s} q_{j} \theta_{j}^{i+1} \beta^{2+m+s}(1+m)^{s}\left(\begin{array}{c}
i \\
m
\end{array}\right)}{i ! s !(1+\beta)^{m+1}\left(\mathrm{e}^{\theta_{j}}-1\right)} \int_{0}^{y} x^{n+s}(1+x)\left[(1+x)+\frac{1}{\beta}\right]^{m} \mathrm{~d} x \\
& =\sum_{s, i=0}^{\infty} \sum_{m=0}^{i} \sum_{r=0}^{m} \gamma_{s, i, m, r}^{*} \int_{0}^{y} x^{n+s}(1+x)^{r+1} \mathrm{~d} x,
\end{aligned}
$$

where $\gamma_{s, i, m, r}^{*}$ is defined above.

Using the MATHEMATICA software for calculating the last integral, the incomplete moments of $X$ are

$$
m_{n}(y)=\sum_{s, i=0}^{\infty} \sum_{m=0}^{i} \sum_{r=0}^{m} \gamma_{s, i, m, r}^{*} \frac{{ }_{2} F_{1}(-1-r, 1+n+s ; 2+n+s ;-y) y^{1+n+s}}{1+n+s}
$$

where ${ }_{2} F_{1}(a, b ; c ; x)=B(b, c-b)^{-1} \int_{0}^{1} \frac{t^{b-1}(1-t)^{c-b-1}}{(1-t x)^{a}} \mathrm{~d} t$ is the well-know hypergeometric function (Gradshteyn and Ryzhik (17)).

An expression for the moment generating function (mgf) of $X$ can be obtained from Equation (10) using the LP density function. We can express it as

$$
M_{X}(t)=\sum_{i=0}^{\infty} \sum_{r=0}^{i} \sum_{s=0}^{r} \gamma_{i, s, r}^{* *}[\beta(1+r)-t]^{-2-s}[1+\beta(1+r)+s-t] \Gamma(1+s)
$$

where

$$
\gamma_{i, s, r}^{* *}=\sum_{j=0}^{\infty} \frac{(-1)^{s} q_{j} \theta_{j}^{i+1} \beta^{s+2}\left(\begin{array}{c}
i \\
r
\end{array}\right)\left(\begin{array}{c}
r \\
s
\end{array}\right)}{i !\left(\mathrm{e}^{\theta_{j}}-1\right)(\beta+1)^{s+1}} .
$$

\subsection{Mean deviations}

The mean deviations about the mean $\left[\delta_{1}(X)=E\left(\left|X-\mu_{1}^{\prime}\right|\right)\right]$ and about the median $\left[\delta_{2}(X)=E(|X-M|)\right]$ of $X$ can be expressed as

$$
\delta_{1}(X)=2 \mu_{1}^{\prime} F\left(\mu_{1}^{\prime}\right)-2 m_{1}\left(\mu_{1}^{\prime}\right) \quad \text { and } \quad \delta_{2}(X)=\mu_{1}^{\prime}-2 m_{1}(M),
$$

respectively, where $\mu_{1}^{\prime}=E(X), M=\operatorname{Median}(X)$ is the median computed from (7) with $u=1 / 2, F\left(\mu_{1}^{\prime}\right)$ is calculated from the cdf $(5)$ and $m_{1}(z)=\int_{-\infty}^{z} x f(x) \mathrm{d} x$ is the first incomplete moment given by (13) with $n=1$.

Setting $u=G(x)$ in (11) gives

$$
m_{1}(z)=\sum_{j=0}^{\infty} q_{j} T_{k}(z)
$$

where the integral $T_{k}(z)$ can be expressed in terms of $Q(u)=G^{-1}(x)$ as

$$
T_{k}(z)=(k+a) c \int_{0}^{G(z)} Q(u) u^{(k+a) c-1} \mathrm{~d} u .
$$


An alternative representation for $m_{1}(z)$ can be derived from (10) as

$$
m_{1}(z)=\int_{-\infty}^{z} x f(x) \mathrm{d} x=\sum_{j=0}^{\infty} q_{j} J_{k}(z)
$$

where

$$
J_{k}(z)=\int_{-\infty}^{z} x h_{(k+a) c}(x) \mathrm{d} x
$$

The Lorenz and Bonferroni curves are important applications of the mean deviations in fields like economics, reliability, demography insurance and medicine. They are defined for a given probability $\pi$ by $B(\pi)=$ $m_{1}(q) /\left(\pi \mu_{1}^{\prime}\right)$ and $L(\pi)=m_{1}(q) / \mu_{1}^{\prime}$ respectively, where $\mu_{1}^{\prime}=E(X)$ and $q=Q(\pi)$ is given by (7). The Bonferroni and Lorenz curves for the McLP distribution as functions of $\pi$ are readily calculated from (13) for $n=1$.

\subsection{Order statistics}

Order statistics make their appearance in many areas of statistical theory and practice. Order statistics play an important role in quality control and reliability, where some predictors are often based on their moments. We derive an explicit expression for the density function of the $i$ th order statistic $X_{i: n}$, say $f_{i: n}(x)$. Suppose $X_{1}, X_{2}, \ldots, X_{n}$ independent and identically distributed random variables from the McLP distribution. Let $X_{i: n}$ denote the $i$ th order statistic. From Equations (4) and (5), the pdf of $X_{i: n}$ can be expressed as an infinite linear combination of LP density functions

$$
f_{i: n}(x)=\sum_{k, r, t=0}^{\infty} \sum_{j=0}^{n-i} p_{k, r, t} g(x ; \beta, \theta(t+1))
$$

where

$$
p_{k, r, t}=\frac{(-1)^{j+t} n ! \omega_{k}(k+a) c d_{j+i-1, r}\left(1-\mathrm{e}^{\theta}\right)^{-c a(j+i)}}{(i-1) !(n-i) !(t+1)\left(1-\mathrm{e}^{\theta}\right)^{c(k+1)-1}}\left(\begin{array}{c}
n-i \\
j
\end{array}\right)\left(\begin{array}{c}
c[k+a(j+1)+1]-1 \\
t
\end{array}\right)
$$

and $d_{j+i-1, r}$ are defined recursively by

$$
d_{j+i-1, r}=\left(r \omega_{0}\right)^{-1} \sum_{m=1}^{r}[m(j+i)-r] \omega_{m} d_{j+i-1, r-m} \text { with } d_{j+i-1,0}=\omega_{0}^{j+i-1} .
$$

Clearly, the cdf of $X_{i: n}$ can be expressed as

$$
F_{i: n}(x)=\sum_{k, r, t=0}^{\infty} \sum_{j=0}^{n-i} p_{k, r, t} G(x ; \beta, \theta(t+1)) .
$$

Equation (14) reveals that the pdf of $X_{i: n}$ can be represented as a finite mixture of Lindley Poisson densities. Thus, some mathematical properties of $X_{i: n}$ can be obtained from (14). For example, the moments and mgf of $X_{i: n}$ are given by

$$
E\left(X_{i: n}^{s}\right)=\sum_{k, r, t=0}^{\infty} \sum_{j=0}^{n-i} p_{k, r, t} E\left(Z^{s}\right)
$$


and

$$
M_{i: n}(t)=\sum_{k, r, t=0}^{\infty} \sum_{j=0}^{n-i} p_{k, r, t} E\left(\mathrm{e}^{t Z}\right)
$$

where $Z \sim \operatorname{LP}(\beta, \theta(t+1))$.

\section{Estimation}

We calculate the maximum likelihood estimates (MLEs) of the parameters of the McLP distribution from complete samples only. Let $x_{1}, \ldots, x_{n}$ be a random sample of size $n$ from the $\operatorname{McLP}(a, b, c, \beta, \theta)$ distribution. The log-likelihood function for the vector of parameters $\boldsymbol{\theta}=(a, b, c, \beta, \theta)^{T}$ can be expressed as

$$
\begin{aligned}
\ell(\boldsymbol{\theta})= & n \log (c)+n \log (\theta)+2 n \log (\beta)-n \log (B(a, b))-n \log (1+\beta)+\sum_{i=1}^{n} \log \left(1+x_{i}\right) \\
& -\beta \sum_{i=1}^{n} x_{i}+\sum_{i=1}^{n} \varphi\left(x_{i}\right)+(a c-1) \sum_{i=1}^{n} \log \left(\mathrm{e}^{\varphi\left(x_{i}\right)}-1\right) \\
& +(b-1) \sum_{i=1}^{n} \log \left[\left(\mathrm{e}^{\theta}-1\right)^{c}-\left(\mathrm{e}^{\varphi\left(x_{i}\right)}-1\right)^{c}\right] .
\end{aligned}
$$

The elements of the score vector are given by

$$
\begin{aligned}
& \frac{\partial \ell}{\partial a}=-\frac{n}{B(a, b)}+c \sum_{i=1}^{n} \log \left(\mathrm{e}^{\varphi\left(x_{i}\right)}-1\right)-n c \log \left(\mathrm{e}^{\theta}-1\right), \\
& \frac{\partial \ell}{\partial b}=-\frac{n}{B(a, b)}+\sum_{i=1}^{n} \log \left[\left(\mathrm{e}^{\theta}-1\right)^{c}-\left(\mathrm{e}^{\varphi\left(x_{i}\right)}-1\right)^{c}\right]-n c \log \left(\mathrm{e}^{\theta}-1\right), \\
& \frac{\partial \ell}{\partial c}=\frac{n}{c}+(b-1) \sum_{i=1}^{n} \frac{\left(\mathrm{e}^{\theta}-1\right)^{c} \log \left(\mathrm{e}^{\theta}-1\right)-\left(\mathrm{e}^{\varphi\left(x_{i}\right)}-1\right)^{c}\left(\mathrm{e}^{\varphi\left(x_{i}\right)}-1\right)}{\left(\mathrm{e}^{\theta}-1\right)^{c}-\left(\mathrm{e}^{\varphi\left(x_{i}\right)}-1\right)^{c}} \\
& +a \sum_{i=1}^{n} \log \left(\mathrm{e}^{\varphi\left(x_{i}\right)}-1\right)-n \log \left(\mathrm{e}^{\theta}-1\right) \\
& \frac{\partial \ell}{\partial \beta}=\frac{2 n}{\beta}-\frac{n}{1+\beta}-\sum_{i=1}^{n} x_{i}+\sum_{i=1}^{n} \frac{\partial}{\partial \beta} \varphi\left(x_{i}\right)+(a c-1) \sum_{i=1}^{n} \frac{\mathrm{e}^{\varphi\left(x_{i}\right)} \frac{\partial}{\partial \beta} \varphi\left(x_{i}\right)}{\mathrm{e}^{\varphi\left(x_{i}\right)}-1} \\
& -c(b-1) \sum_{i=1}^{n} \frac{\mathrm{e}^{\varphi\left(x_{i}\right)}\left(\mathrm{e}^{\varphi\left(x_{i}\right)}-1\right)^{c-1} \frac{\partial}{\partial \beta} \varphi\left(x_{i}\right)}{\left(\mathrm{e}^{\theta}-1\right)^{c}-\left(\mathrm{e}^{\varphi\left(x_{i}\right)}-1\right)^{c}}, \\
& \frac{\partial \ell}{\partial \theta}=\frac{n}{\theta}+\sum_{i=1}^{n} V\left(x_{i}\right)-\frac{n c(a+b-1) \mathrm{e}^{\theta}}{\mathrm{e}^{\theta}-1}+(a c-1) \sum_{i=1}^{n} \frac{V\left(x_{i}\right) \mathrm{e}^{\varphi\left(x_{i}\right)}}{\mathrm{e}^{\varphi\left(x_{i}\right)}-1} \\
& +c(b-1) \sum_{i=1}^{n} \frac{\mathrm{e}^{\theta}\left(\mathrm{e}^{\theta}-1\right)^{c-1}-V\left(x_{i}\right) \mathrm{e}^{\varphi\left(x_{i}\right)}\left(\mathrm{e}^{\varphi\left(x_{i}\right)}-1\right)^{c-1}}{\left(\mathrm{e}^{\theta}-1\right)^{c}-\left(\mathrm{e}^{\varphi\left(x_{i}\right)}-1\right)^{c}} .
\end{aligned}
$$


Note that since $\varphi(x)=\theta\left[1-\left(1+\frac{\beta x}{1+\beta}\right) \mathrm{e}^{-\beta x}\right]$, we have

$$
\frac{\partial \varphi}{\partial \beta}=\theta \mathrm{e}^{-\beta x}\left[\left(1+\frac{\beta x}{1+\beta}\right)-\frac{1}{(1+\beta)^{2}}\right]
$$

and

$$
\frac{\partial \varphi}{\partial \theta}=\left[1-\left(1+\frac{\beta x}{1+\beta}\right) \mathrm{e}^{-\beta x}\right]=V(x)
$$

The maximum likelihood estimates, $\widehat{\boldsymbol{\theta}}$ of $\boldsymbol{\theta}=(a, b, c, \beta, \theta)$ are obtained by solving the nonlinear equations $\frac{\partial \ell}{\partial a}=0, \frac{\partial \ell}{\partial b}=0, \frac{\partial \ell}{\partial c}=0, \frac{\partial \ell}{\partial \beta}=0, \frac{\partial \ell}{\partial \theta}=0$. These equations are not in closed form and the values of the parameters $a, b, c, \beta$ and $\theta$, must be found by using iterative methods. We maximize the likelihood function using NLmixed procedure in SAS as well as the function nlm in R. These functions were applied and executed for a wide range of initial values. This process often results or leads to more than one maximum, however, in these cases, we take the MLEs corresponding to the largest value of the maxima. In a few cases, no maximum was identified for the selected initial values. In these cases, a new initial value was tried in order to obtain a maximum. The issues of existence and uniqueness of the MLEs are of theoretical interest and have been studied by several authors for different distributions (Seregin (18); Santos Silva and Tenreyro (19); Xia et al. (20); Zhou (21)). At this point we are not able to address the theoretical aspects (existence, uniqueness) of the MLE of the parameters of the McLP distribution.

\section{A simulation study}

To examine the performance of the MLEs for the McLP distribution, we perform a simulation study:

1. generate $r$ samples of size $n$ from (4) using (7).

2. compute the MLEs for the $r$ samples, say $\left(\widehat{a_{i}}, \widehat{b_{i}}, \widehat{c_{i}}, \widehat{\beta}_{i}, \widehat{\theta}_{i}\right)$ for $i=1, \ldots, r$.

3. compute the standard errors of the MLEs for $r$ samples, say $\left(s_{\widehat{a}}, s_{\widehat{b}}, s_{\widehat{c}}, s_{\widehat{\beta}}, s_{\widehat{\theta}}\right)$ for $i=1, \ldots, r$. The standard errors are computed by inverting the observed information matrices.

4. compute the average biases (bias) and root mean squared errors (RMSE) by

$$
\operatorname{bias}_{\epsilon}(n)=\frac{1}{r} \sum_{i=1}^{r}\left(\widehat{\epsilon}_{i}-\epsilon\right) \text { and } \operatorname{RMSE}_{\epsilon}(n)=\sqrt{\frac{1}{r} \sum_{i=1}^{r}\left(\widehat{\epsilon}_{i}-\epsilon\right)^{2}}
$$

for $\epsilon=a, b, c, \beta, \theta$.

We repeat these steps for $r=1,000$ values and $n=50,100, \ldots, 500$ with $a=4.0, b=5.0, c=1.5, \beta=2.3$ and $\theta=7.0$, so computing $\operatorname{bias}_{\epsilon}(n)$ and $\operatorname{RMSE}_{\epsilon}(n)$. Table 1 presents the bias and RMSE values. 
Table 1: Simulation results: average bias and RMSE.

\begin{tabular}{c|ccccc|cccccc}
\hline \multirow{2}{*}{$n$} & \multicolumn{4}{|c|}{ bias } & \multicolumn{6}{c}{ RMSE } \\
\cline { 2 - 12 } & $\widehat{a}$ & $\widehat{b}$ & $\widehat{c}$ & $\widehat{\beta}$ & $\widehat{\theta}$ & $\widehat{a}$ & $\widehat{b}$ & $\widehat{c}$ & $\widehat{\beta}$ & $\widehat{\theta}$ \\
\hline 30 & 12.656 & 14.2343 & 25.432 & 0.631 & 19.992 & 1021.11 & 4412.321 & 2991.110 & 2.929 & 2743.5407 \\
50 & 12.449 & 15.209 & 26.054 & 0.600 & 20.794 & 1018.055 & 4106.483 & 2927.303 & 2.396 & 2668.257 \\
100 & 5.375 & 3.499 & 14.586 & 0.373 & 13.517 & 192.646 & 329.193 & 908.035 & 1.045 & 816.219 \\
150 & 4.401 & 1.817 & 9.053 & 0.232 & 12.702 & 79.541 & 62.149 & 349.803 & 0.648 & 358.731 \\
200 & 3.645 & 1.337 & 10.170 & 0.212 & 11.561 & 51.626 & 37.535 & 363.347 & 0.520 & 333.503 \\
250 & 4.449 & 1.915 & 10.686 & 0.102 & 5.495 & 59.118 & 43.648 & 262.881 & 0.505 & 186.046 \\
300 & 5.205 & 2.402 & 11.047 & 0.009 & 2.279 & 70.781 & 49.030 & 216.976 & 0.500 & 126.430 \\
350 & 4.160 & 1.076 & 9.780 & 0.124 & 7.496 & 56.147 & 23.167 & 271.300 & 0.386 & 210.205 \\
400 & 4.225 & 0.934 & 8.610 & 0.107 & 7.371 & 56.689 & 19.940 & 216.844 & 0.329 & 171.200 \\
450 & 4.383 & 0.806 & 8.525 & 0.095 & 8.742 & 60.682 & 14.475 & 232.612 & 0.307 & 207.301 \\
500 & 4.028 & 0.550 & 7.973 & 0.119 & 10.727 & 54.439 & 11.115 & 226.542 & 0.280 & 226.675 \\
\hline
\end{tabular}

The biases of the estimates decrease when $n \rightarrow \infty$. The reported results hold only for the choice $(a, b, c, \beta, \theta)=$ $(4.0,5.0,1.5,2.3,7.0)$. However, the results are similar for a wide range of this parameter vector. We also observe that as the sample size $n$ increases, the RMSEs likewise decreases.

\section{Application}

In this section, we present two applications of the McLP model to the data obtained from Bader and Priest (22) (data set I) and Bjerkedal (23) (data set II). The data set I consist of 56 strength data measured in GPA, the single carbon fibers and impregnated 1000-carbon fiber tows. Single fibers were tested under tension at gauge length $1 \mathrm{~mm}$. The second real data set represents the survival times (in days) of 72 guinea pigs infected with virulent tubercle bacilli. In Table 2 we provide some descriptive statistics for both sets of data.

Table 2: Descriptive Statistics for the data sets I and II

\begin{tabular}{cccccccccc}
\hline Data sets & Min. & Mean & Median & s.d. & Skewness & Kurtosis & $1^{\text {st }} Q u$. & $3^{r d} Q u$. & Max. \\
\hline \hline I & 2.247 & 4.261 & 4.248 & 0.821 & 0.072 & -0.211 & 3.728 & 4.683 & 6.060 \\
II & 0.100 & 1.851 & 1.560 & 1.201 & 1.788 & 4.157 & 1.080 & 2.303 & 7.000 \\
\hline \hline
\end{tabular}

The total time on test (TTT) plot proposed by Aarset (24) is a means to check the shape of the observed hazard function. This is drawn by plotting $T(i / n)=\left[\sum_{r=1}^{i} y_{(r)}+(n-i) y_{(r)}\right] / \sum_{r=1}^{i} y_{(r)}$, where, $i=$ $1,2, \ldots, n$ and $y_{(r)}(r=1,2, \ldots, n)$ is the order statistics of the sample, against $i / n$. For constant hazard plot is a straight diagonal while for decreasing (increasing) hazards it is convex (concave) respectively. The TTT plots for the data sets (Figure 3) indicate that all the data sets have increasing hazard rate. 

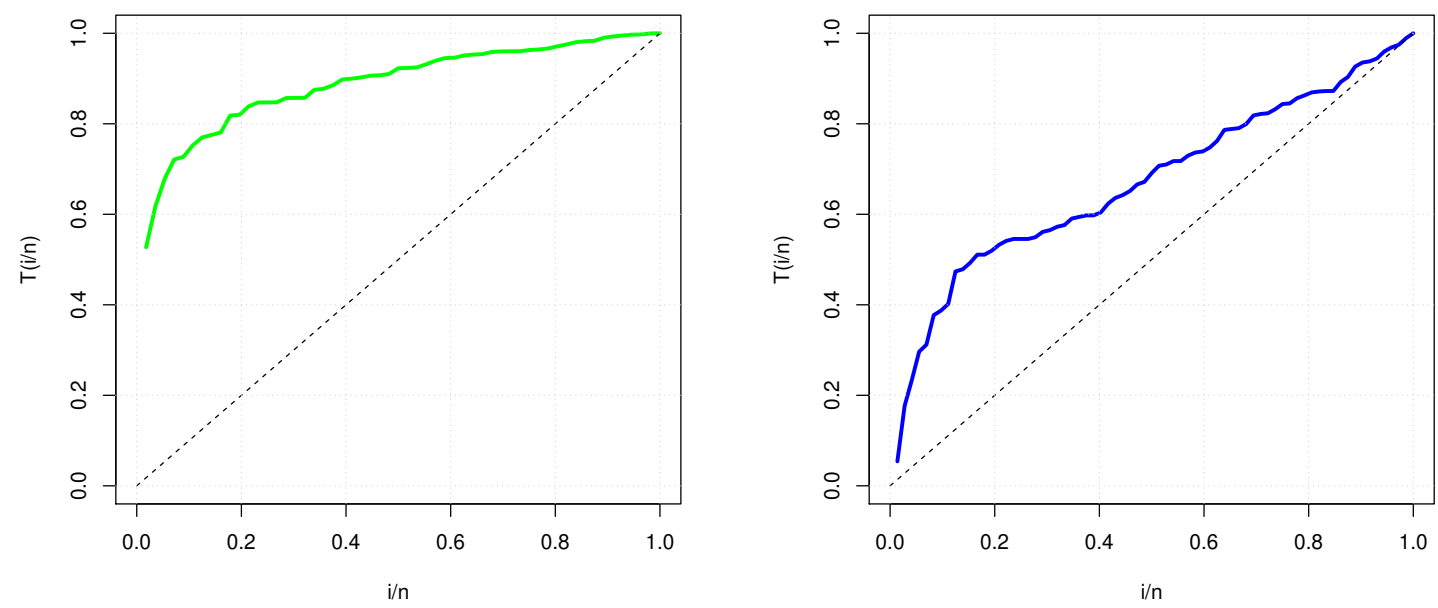

Figure 3: TTT plots of the data sets I and II from left to right

We present the fits of the LP, ELP, BLP, KwLP and McLP distributions. Estimates of the parameters of the distributions and standard errors (SEs) of the MLEs of the parameters for each competing models are obtained in Table 3.

Table 3: MLEs, standard errors (in parentheses) and confidence intervals (in parentheses) values for the data sets I and II

\begin{tabular}{l|ccccc}
\hline Distribution & $\widehat{a}$ & $\widehat{b}$ & $\widehat{c}$ & $\widehat{\beta}$ & $\widehat{\theta}$ \\
\hline Data set I & & & & & \\
\hline LP & - & - & - & 1.441 & 79.963 \\
& & & & $(0.123)$ & $(17.134)$ \\
ELP & - & - & 4.541 & 1.440 & 17.555 \\
& & & $(0.992)$ & $(0.123)$ & $(2.935)$ \\
BLP & 2.265 & 4.752 & - & 0.596 & 7.466 \\
& $(0.614)$ & $(1.061)$ & - & $(0.687)$ & $(1.733)$ \\
KwLP & - & 6.936 & 3.621 & 0.726 & 5.066 \\
& - & $(1.316)$ & $(0.886)$ & $(0.379)$ & $(1.516)$ \\
McLP & 0.504 & 5.184 & 9.459 & 0.868 & 4.307 \\
& $(0.374)$ & $(2.593)$ & $(2.317)$ & $(0.240)$ & $(1.443)$ \\
\hline Data set II & & & & & \\
\hline LP & - & - & - & 1.525 & 4.302 \\
& & & & $(0.146)$ & $(0.990)$ \\
ELP & - & - & 2.949 & 0.356 & 7.985 \\
& & & $(0.912)$ & $(0.051)$ & $(1.235)$ \\
BLP & 0.459 & 0.468 & - & 2.324 & 13.876 \\
& $(0.014)$ & $(0.061)$ & - & $(0.109)$ & $(2.013)$ \\
KwLP & - & 0.422 & 0.739 & 2.561 & 8.295 \\
& - & $(0.156)$ & $(0.666)$ & $(0.596)$ & $(1.516)$ \\
McLP & 0.281 & 0.556 & 4.561 & 1.980 & 4.345 \\
& $(0.033)$ & $(0.106)$ & $(0.181)$ & $(0.182)$ & $(1.032)$ \\
\hline
\end{tabular}

We have considered known model selection criteria namely the AIC (Akaike Information Criterion), BIC (Bayesian Information Criterion), CAIC (Consistent Akaike Information Criterion) and HQIC (HannanQuinn Information Criterion) and Anderson-Darling ( $\left.\mathrm{A}^{*}\right)$ and Cramér-von Mises ( $\left.\mathrm{W}^{*}\right)$ goodness-of-fit statistics to compare the fitted models (Table 4 ). The statistics $\mathrm{A}^{*}$ and $\mathrm{W}^{*}$ are described by Chen and Balakrishnan (25). In general, the smaller the values of these statistics, the better the fit to the data. 
Table 4: AIC, BIC, CAIC, HQIC, $\mathrm{A}^{*}$ and $\mathrm{W}^{*}$ values for the data sets I and II

\begin{tabular}{|c|c|c|c|c|c|c|}
\hline Distribution & $\mathrm{AIC}$ & $\mathrm{BIC}$ & CAIC & HQIC & $\mathrm{A}^{*}$ & $\mathrm{~W}^{*}$ \\
\hline \multicolumn{7}{|l|}{ Data set I } \\
\hline $\mathrm{LP}$ & 149.96 & 154.02 & 150.19 & 151.52 & 0.61 & 0.09 \\
\hline ELP & 148.36 & 154.43 & 148.82 & 150.71 & 0.53 & 0.08 \\
\hline BLP & 145.90 & 154.02 & 146.68 & 149.02 & 0.36 & 0.08 \\
\hline KwLP & 145.78 & 153.90 & 146.56 & 148.90 & 0.28 & 0.07 \\
\hline McLP & 141.74 & 151.84 & 142.94 & 145.64 & 0.17 & 0.02 \\
\hline \multicolumn{7}{|l|}{ Data set II } \\
\hline LP & 205.74 & 210.28 & 205.91 & 207.54 & 0.85 & 0.14 \\
\hline ELP & 205.13 & 210.96 & 204.49 & 206.85 & 0.53 & 0.07 \\
\hline BLP & 204.78 & 213.88 & 205.37 & 208.40 & 0.46 & 0.07 \\
\hline KwLP & 204.86 & 213.96 & 205.45 & 208.48 & 0.44 & 0.06 \\
\hline McLP & 198.48 & 209.83 & 199.38 & 202.98 & 0.40 & 0.05 \\
\hline
\end{tabular}

Table 5: LR statistics for the data sets I and II

\begin{tabular}{cccc}
\hline Model & Hypothesis & $\begin{array}{c}\text { LR } \\
(p \text {-value })\end{array}$ & $\begin{array}{c}\text { LR } \\
(p \text {-value })\end{array}$ \\
\hline data set & & I & II \\
\hline LP vs. McLP & $\mathcal{H}_{0}: a=b=c=1$ vs. $\mathcal{H}_{1}: \mathcal{H}_{0}$ is false & 14.22 & 13.26 \\
& & $(0.002)$ & $(0.004)$ \\
ELP vs. McLP & $\mathcal{H}_{0}: a=b=c=1$ vs. $\mathcal{H}_{1}: \mathcal{H}_{0}$ is false & 10.60 & 9.64 \\
& $\mathcal{H}_{0}: c=1$ vs. $\mathcal{H}_{1}: c \neq 1$ & $(0.004)$ & $(0.008)$ \\
BLP vs. McLP & $\mathcal{H}_{0}: a=1$ vs. $\mathcal{H}_{1}: a \neq 1$ & 6.16 & 8.28 \\
KwLP vs. McLP & & $6.01)$ & $(0.004)$ \\
& & $(0.02)$ & 8.38 \\
& & & $(0.003)$ \\
\hline
\end{tabular}

Let $\boldsymbol{\theta}=(a, b, c, \beta, \theta)$ be a parametric vector, the LR test statistic is given by LR $=-2\left[\ell\left(\hat{\boldsymbol{\theta}}^{*} ; x\right)-\ell(\hat{\boldsymbol{\theta}} ; x)\right]$ where $\hat{\boldsymbol{\theta}}^{*}$ is the restricted MLEs under the null hypothesis $\mathcal{H}_{0}$ and $\hat{\boldsymbol{\theta}}$ is the unrestricted MLEs estimates under the alternative hypothesis $\mathcal{H}_{1}$. Under the null hypothesis, the LR criterion follows Chi-square distribution. The null hypothesis can not be accepted for p-value less than 0.05. A comparison of the McLP distribution with some of its nested models using LR statistics is performed in Table 5.

The Tables 4 and 5 indicate that the fitted McLP distribution to these data is superior to the other fitted models. The plots of the fitteds pdf and cdf of the McLP distribution are given in Figures 4 and 5 . 

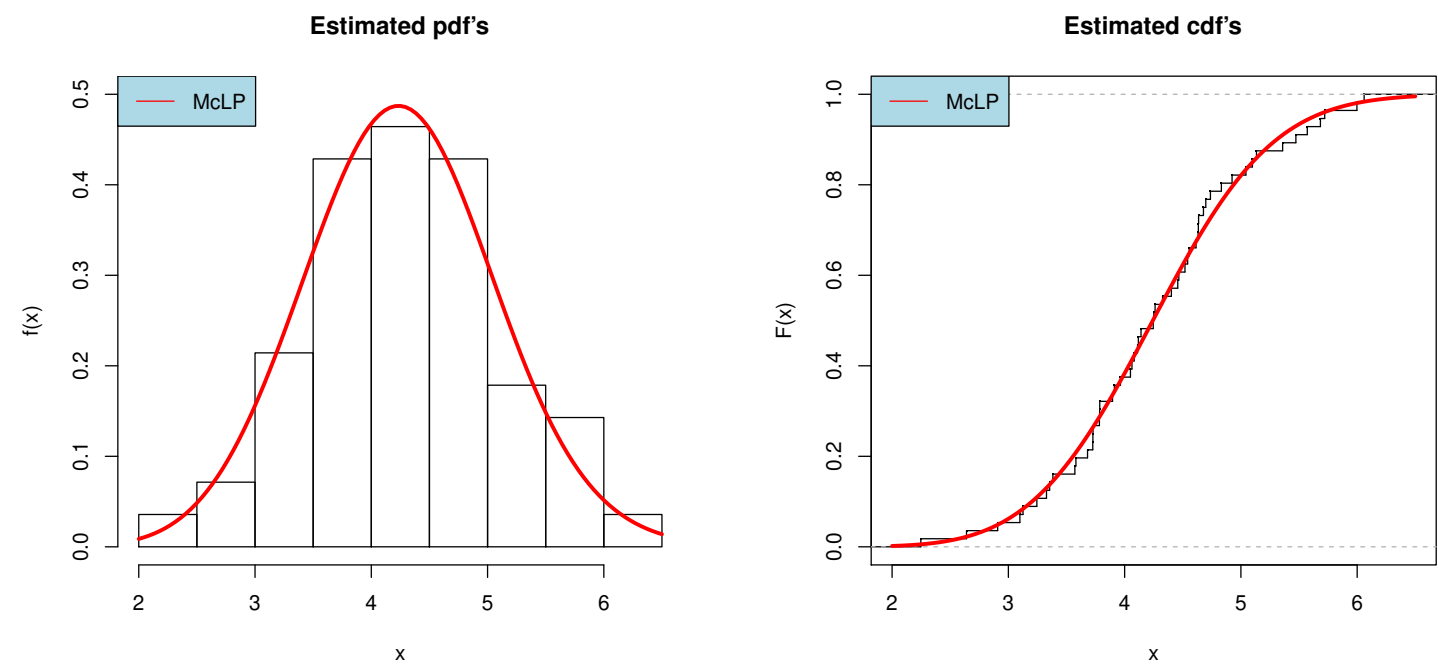

Figure 4: Plots of the observed histogram and estimated pdf and observed ogive and estimated cdf for the McLP distribution for data I
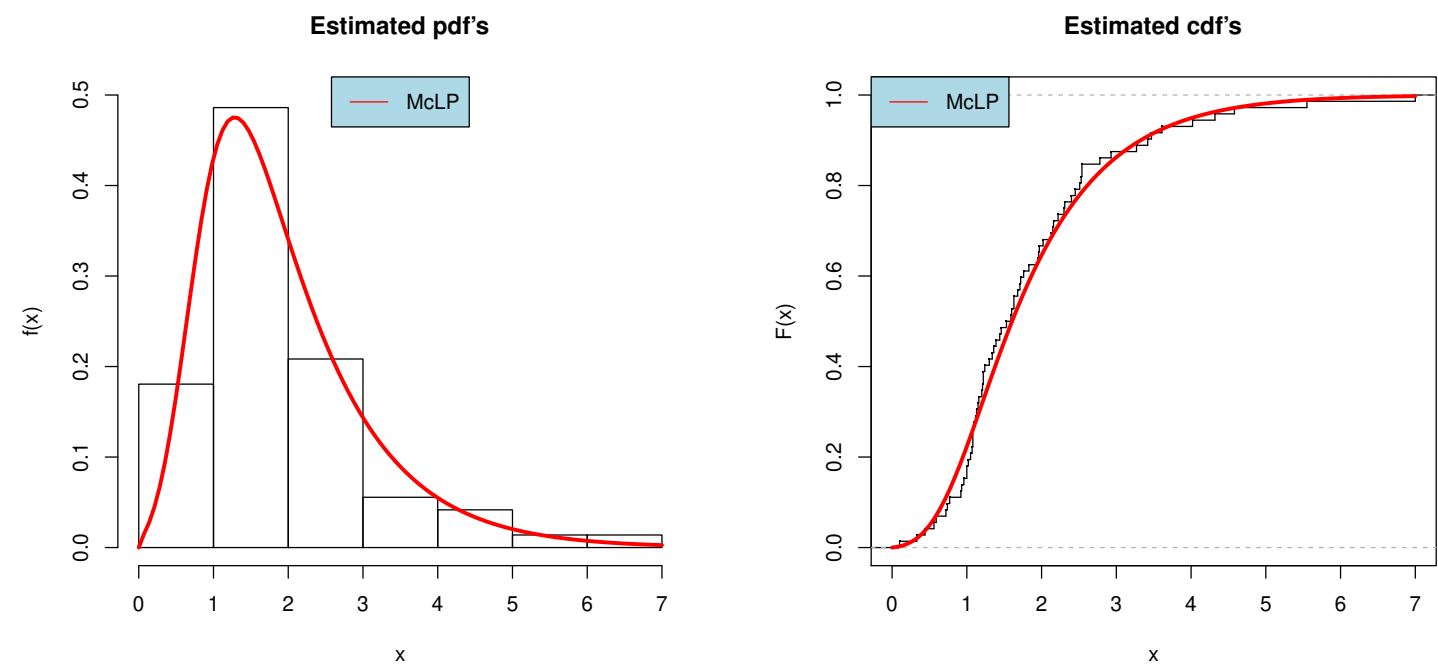

Figure 5: Plots of the observed histogram and estimated pdf and observed ogive and estimated cdf for the McLP distribution for data II

\section{Conclusions}

The Lindley-Poisson distribution is commonly used to model the lifetime of a system. However, it does not exhibit a bathtub-shaped failure rate function and thus it can not be used to model the complete lifetime of a system. We define a new lifetime model, called the McDonald Lindley-Poisson distribution, which extends the Kumaraswamy Lindley-Poisson distribution proposed by Pararai et al. (11), whose failure rate function can be increasing, decreasing and bathtub. The McDonald Lindley-Poisson density function can be expressed as a linear combination of Lindley-Poisson densities, which allows to obtain several of its structural properties. We provide a mathematical treatment of the distribution including explicit expressions for the density function, generating function, ordinary and incomplete moments, generating function, quantile function, mean deviations and order statistics and their moments. The parameter estimation is approached by maximum likelihood and the observed information matrix is derived. The usefulness of the new model is illustrated in applications to real data using formal goodness-of-fit tests. By means of two real data sets 
application, we show that the proposed distribution can give a better fits them other competitive models.

\section{References}

1. Minka T.P., Shmueli G., Kadane J.B., Borle S. and Boatwright P. (2003). Computing with the COM-Poisson distribution. Technical Report: 776, Department of Statistics, Carnegie Mellon University, http://www.stat.cmu.edu/tr/tr776/tr776.html.

2. Shmueli,G., Minka, T.P., Kadane, J.B., Borle, S. and Boatwright, P. (2005). A useful distribution for fitting discrete data: revival of the Conway-Maxwell-Poisson distribution. Journal of the Royal Statistical Society Series C, 54, 127-142.

3. Kus, C. (2007). A new lifetime distribution. Computational Statistics \& Data Analysis, 51, 44974509.

4. Hemmati, F. and Khorram, E. (2011). A new three-parameter ageing distribution. Journal of Statistical Planning and Inference, 141, 2266-2275.

5. Silva, R.V., Gomes-Silva, F., Ramos, M.W.A. and Cordeiro,G.M. (2014). The exponentiated Burr XII Poisson distribution with application to lifetime data. International Journal of Statistics and Probability, 4, 112-131.

6. Lindley, D.V. (1958). Fiducial distributions and Bayes' Theorem. Journal of the Royal Statistical Society Series B, 20, 102-107.

7. Ghitany, M.E., Atieh, B. and Nadarajah, S. (2008). Lindley distribution and its applications. Mathematics and Computers in Simulation, 78, 493-506.

8. Sankaran, M. (1970). The discrete Poisson-Lindley distribution. Biometrics, 26, 145-149.

9. Mahmoudi E. and Zakerzadeh H. (2010). Generalized Poisson-Lindley distribution. Communications in Statistics - Theory and Methods, 39, 1785-1798.

10. Bhati, D., Sastry, D.V.S. and Maha Qadri, P.Z. (2015). A New Generalized Poisson-Lindley Distribution: Applications and Properties. Austrian Journal of Statistics, 44, 35-51.

11. Pararai, M., Oluyede, B.O. and Warahena-Liyanage, G. (2015). Kumaraswamy Lindley-Poisson distribution: theory and applications. Asian Journal of Mathematics and Applications, 2015: $1-30$.

12. Alexander, C., Cordeiro, G.M., Ortega, E.M.M. and Sarabia, J.M. (2012). Generalized betagenerated distributions. Computational Statistics and Data Analysis, 56, 1880-1897.

13. Eugene, N., Lee, C. and Famoye, F. (2002). Beta-normal distribution and its applications. Communications in Statistics - Theory and Methods, 31, 497-512.

14. Cordeiro, G.M. and de Castro, M. (2010). A New family of generalized distributions. Journal of Statistical Computation and Simulations, 81, 883-898.

15. Gui, W, Zhang, S, Lu, X. (2014). The Lindley-Poisson distribution in lifetime analysis and its properties. Hacettepe Journal of Mathematics and Statistics, 43, 1063-1077.

16. Corless, R.M., Gonnet, G.H., Hare, D.E.G., Jeffrey, D.J. and Knuth, D.E. (1996). On the Lambert W function. Advances in Computational Mathematics, 5, 329-359.

17. Gradshteyn, I.S. Ryzhik, I.M. (2007). Table of integrals, series, and products, Academic Press, New York.

18. Seregin, A. (2010). Uniqueness of the maximum likelihood estimator for k-monotone densities, Proceedings of the American Mathematical Society, 138, 4511-4515.

19. Santos Silva J. M. C., Tenreyro, S. (2010). On the Existence of Maximum Likelihood Estimates in Poisson Regression. Economics Letters, 107, 310-312.

20. Xia, J., Mi, J., Zhou, Y.Y. (2009). On the existence and uniqueness of the maximum likelihood estimators of normal and log-normal population parameters with grouped data, Journal of Probability and Statistics, Article id 310575:16 pages.

21. Zhou, C. (2009). Existence and consistency of the maximum likelihood estimator for the extreme index, J. Multivariate Analysis, 100, 794-815.

22. Bader, M. and Priest, A. (1982) Statistical aspects of fibre and bundle strength in hybrid composites, Progress in science and engineering of composites, Tokyo.

23. Bjerkedal, T. (1960). Acquisition of resistance in guinea pigs infected with different doses of virulent tubercle bacilli. American Journal Hygenes, 72, 130-148. 
24. Aarset, M.V. (1987). How to identify a bathtub hazard rate. IEEE Transactions on Reliability, 36, 106-108.

25. Chen, G. and Balakrishnan, N. (1995). Ageneral purpose approximate goodness-of-fit test. Journal of Quality Technology, 27, 154-161. 